Pacific Journal of Mathematics

MATRIX SUMMABILITY OVER CERTAIN CLASSES O
SEQUENCES ORDERED WITH RESPECT TO RATE OF

David Fleming Dawson 


\title{
MATRIX SUMMABILITY OVER CERTAIN CLASSES OF SEQUENCES ORDERED WITH RESPECT TO RATE OF CONVERGENCE
}

\author{
DAVID F. DAWSON
}

Let $C_{0}$ denote the set of all complex null sequences, and let $S_{0}$ denote the set of all sequences in $C_{0}$ which have at most a finite number of zero terms. If $a=\left\{a_{p}\right\} \in S_{0}$ and $b=\left\{b_{p}\right\} \in S_{0}$, we say that $a$ converges faster than $b, a<b$, provided $\lim a_{p} / b_{p}=0$. We say that $a$ and $b$ converge at the same rate, $a \sim b$, provided $0<\lim \inf \left|a_{p} / b_{p}\right|$ and $\lim \sup \left|a_{p} / b_{p}\right|<\infty$. If $a \in S_{0}$, let $[a]=\left\{x \in S_{0}: x \sim a\right\}$. Let $E_{0}=\left\{[x]: x \in S_{0}\right\}$. If $[a],[b] \in E_{0}$, then we say that $[a]$ is less than $[b],[a]<^{\prime}[b]$, provided $a<b$. We note that $E_{0}$ is partially ordered with respect to $\leqq \prime$. In this paper we study matrix summability over subsets of $S_{0}$ and over elements of $E_{0}$. Open intervals in $S_{0}$ will be denoted by $(a, b),(a,-)$, and $(-, b)$, where $(a,-)=\left\{x \in S_{0}: a<x\right\}$ and $(-, b)=\left\{x \in S_{0}: x<b\right\}$. Some of our results characterize, for matrices, maximal summability intervals in $S_{0}$. Such intervals are of the form $(-, b)$, never of the form $(-, b]=\left\{x \in S_{0}\right.$ : either $x<b$ or $\left.x \sim b\right\}$.

Notational conveniences used are as follows. If $A=\left(a_{p q}\right)$ is a matrix and $b$ is a sequence such that for each positive integer $p$, the series $\sum_{q=1}^{\infty} a_{p q} b_{q}$ converges, then $A(b)$ will denote the sequence $\left\{\sum_{q=1}^{\infty} a_{p q} b_{q}\right\}_{p=1}^{\infty}$. We will use $A_{b}$ to denote the matrix $\left(a_{p q} b_{q}\right)$. If each of $a$ and $b$ is a sequence, then $a b$ will be used to denote the sequence $\left\{a_{p} b_{p}\right\}$.

Playing a basic role throughout the paper are the two classical Silverman-Toeplitz (abbreviated $S-T$ ) conditions which are necessary and sufficient for a matrix $A$ to be convergence preserving over (abbreviated c.p.o.) $C_{0}$. These conditions are

and

(1) $\left\{a_{p q}\right\}_{p=1}^{\infty}$ converges, $q=1,2,3, \cdots$,

(2) there exists $K$ such that $\sum_{q=1}^{\infty}\left|a_{p q}\right|<K, p=1,2,3, \cdots$. We note that the $S-T$ conditions are necessary and sufficient for a matrix $A$ to be c.p.o. $S_{0}$.

REMARK 1. A matrix sums every sequence in some interval $(-, b)$ if and only if it has convergent columns.

REMARK 2. If the matrix $A$ is c.p.o. [b] and $c$ is a sequence such that $\lim c_{p} / b_{p}=0$, then $A(c)$ is convergent.

Remark 2'. If $A$ is c.p.o. [b], then $A$ is c.p.o. $(-, b]$. 
Remark 3. If $A$ is c.p.o. $(a,-)$, then $A$ is c.p.o. $C_{0}$.

Lemma. Suppose $K$ and $L$ are countable subsets of $S_{0}$ such that if $x \in K$ and $y \in L$, then $x<y$. Then there exists $z \in S_{0}$ such that if $x \in K$ and $y \in L$, then $x<z<y$.

Proof. Our proof will be for the case that both $K$ and $L$ are infinite sets. Let $K=\left\{a^{(1)}, a^{(2)}, a^{(3)}, \cdots\right\}$ and $L=\left\{b^{(1)}, b^{(2)}, b^{(3)}, \cdots\right\}$. Let $\left\{n_{p}\right\}_{p=1}^{\infty}$ be an increasing sequence of positive integers such that if $i>n_{p}$, then

$$
\left|\frac{b_{i}^{(j)}}{a_{i}^{(t)}}\right|>2^{p}, \quad j, t=1,2, \cdots, p .
$$

Define

$$
\begin{aligned}
& c_{i}= b_{i}^{(1)}, i=1,2, \cdots, n_{2}, \\
& c_{i}=(1 / p) \min \left[\left|b_{i}^{(1)}\right|,\left|b_{i}^{(2)}\right|, \cdots,\left|b_{i}^{(p)}\right|\right], \\
& \quad n_{p}<i \leqq n_{p+1}, p=2,3,4, \cdots .
\end{aligned}
$$

Let $r$ be a positive integer. If $p>r$ and $q$ is a positive integer such that $n_{p}<q \leqq n_{p+1}$, then we have $\left|b_{q}^{(r)} / c_{q}\right| \geqq p$, and, since $c_{q}=$ $\left|b_{q}^{(t)}\right| / p$ for some $t \in\{1,2, \cdots, p\}$, we have $\left|c_{q} / a_{q}^{(r)}\right|>2^{p} / p$. Thus $a^{(r)}<c<b^{(r)}$. This completes the proof.

THEOREM 1. If $A$ is c.p.o. [b], then there exists $b^{\prime} \in S_{0}$ such that $b<b^{\prime}$ and $A$ is c.p.o. [ $\left.b^{\prime}\right]$.

Proof. Since $A$ is c.p.o. [b], then by Remarks 1 and 2 ', $A$ has convergent columns. Let $a_{q}=\lim _{p \rightarrow \infty} a_{p q}$. By Remark 2, $A$ sums every null sequence $x$ such that $\lim x_{p} / b_{p}=0$. Thus $A_{b}$ sums every null sequence. Therefore from (2) of the $S-T$ conditions there exists $M$ such that if $n$ is a positive integer, then $\sum_{p=1}^{\infty}\left|a_{n p} b_{p}\right|<M$. Clearly $\sum_{q=1}^{\infty}\left|a_{q} b_{q}\right| \leqq M$. Let $C=\left(c_{p q}\right)$ be the matrix defined by $c_{p q}=a_{p q} b_{q}-a_{q} b_{q}$. Let $D=\left(d_{p q}\right)$ be the matrix defined by $d_{p q}=a_{q} b_{q}$. Then $A_{b}=C+D$. We wish to show that the sequence

$$
\left\{\sum_{p=1}^{\infty}\left|a_{n p} b_{p}-a_{p} b_{p}\right|\right\}_{n=1}^{\infty}
$$

converges to zero. We note that $\left(^{*}\right)$ is bounded. Suppose $\left({ }^{*}\right)$ has a subsequence which converges to $\mu>0$. Note that each column of $C$ converges to zero. Let $n_{1}$ be a positive integer such that

$$
\left|\sum_{p=1}^{\infty}\right| c_{n_{1} p}|-\mu|<\mu / 8 .
$$


Let $k_{1}$ be a positive integer such that $\sum_{p=1}^{k_{1}}\left|c_{n_{1} p}\right|>7 \mu / 8$. Let $N_{1}>n_{1}$ be an integer such that if $q>N_{1}$, then $\sum_{p=1}^{k_{1}}\left|c_{q p}\right|<\mu / 8$. Let $n_{2}>N_{1}$ be an integer such that

$$
\left|\sum_{p=1}^{\infty}\right| c_{n_{2} p}|-\mu|<\mu / 8
$$

Let $k_{2}>k_{1}$ be an integer such that $\sum_{p=1}^{k_{2}}\left|c_{n_{2} p}\right|>7 \mu / 8$. Let $N_{2}>n_{2}$ be an integer such that if $q>N_{2}$, then $\sum_{p=1}^{k_{2}}\left|c_{q p}\right|<\mu / 8$. Continue the process to obtain increasing sequences $\left\{n_{p}\right\}_{p=1}^{\infty}$ and $\left\{k_{p}\right\}_{p=1}^{\infty}$ of positive integers. Define $t_{p q}=\left|c_{p q}\right| / c_{p q}$ if $c_{p q} \neq 0, t_{p q}=1$ if $c_{p q}=0$. Define

$$
\begin{aligned}
& s_{p}=1, p=1,2, \cdots, k_{1}, \\
& s_{p}=(-1)^{q+3} t_{n_{q^{p}}}, k_{q-1}<p \leqq k_{q}, q=2,3,4, \cdots .
\end{aligned}
$$

Suppose $q$ is a positive even integer. Then

$$
\begin{aligned}
& \left|\sum_{p=1}^{\infty} c_{n_{q} p} s_{p}-(-\mu)\right| \\
& =\left|\sum_{p=1}^{k q-1} c_{n_{q} p} s_{p}+\sum_{p=k_{q-1}+1}^{k_{q}} c_{n_{q} p} s_{p}+\sum_{p=k_{q}+1}^{\infty} c_{n_{q} p} s_{p}+\mu\right| \\
& \quad \leqq \sum_{p=1}^{k_{q}-1}\left|c_{n_{q} p}\right|+\sum_{p=k_{q}+1}^{\infty}\left|c_{n_{q} p}\right|+\left|\sum_{p=k_{q-1}+1}^{k_{q}} c_{n_{q} p^{p}} s_{p}+\mu\right| \\
& \quad<\mu / 8+\mu / 4+\left|-\sum_{p=k_{q-1}+1}^{k_{q}}\right| c_{n_{q} p}|+\mu| \\
& \quad<\mu / 8+\mu / 4+\mu / 4 .
\end{aligned}
$$

Similarly, if $q$ is a positive odd integer, then

$$
\left|\sum_{p=1}^{\infty} c_{n_{q} p} s_{p}-\mu\right|<5 \mu / 8
$$

Thus $C(s)$ is divergent. But $A_{b}(s)$ is convergent since $A_{b}(s)=A(b s)$ and $b s \in[b]$. Clearly $D(s)$ is convergent. Hence $C(s)$ is convergent since $C(s)=A_{b}(s)-D(s)$. Therefore we have a contradiction. Thus $\left({ }^{*}\right)$ converges to zero since the assumption to the contrary leads to a contradiction.

Let $j_{1}$ be a positive integer such that if $q>j_{1}$, then $\sum_{p=1}^{\infty}\left|c_{p q}\right|<$ 1/4. Let $K$ be a number such that $\sum_{p=1}^{\infty}\left|c_{n p}\right|<K, n=1,2,3, \cdots$. Let $i_{1}$ be a positive integer such that $\sum_{p=i_{1}+1}^{\infty}\left|c_{n p}\right|<1 / 4, n=1,2, \cdots$, $j_{1}$. Let $j_{2}>j_{1}$ be an integer such that if $q>j_{2}$, then $\sum_{p=1}^{\infty}\left|c_{q p}\right|<1 / 4^{2}$. Let $i_{2}>i_{1}$ be an integer such that $\sum_{p=i_{2}+1}^{\infty}\left|c_{n p}\right|<1 / 4^{2}, n=1,2, \cdots, j_{2}$. Continue the process to obtain increasing sequences $\left\{j_{p}\right\}_{p=1}^{\infty}$ and $\left\{i_{p}\right\}_{p=1}^{\infty}$ of positive integers. Define 


$$
\begin{aligned}
& e_{n}=1, n=1,2, \cdots, i_{1}, \\
& e_{n}=2^{t}, i_{t}<n \leqq i_{t+1}, t=1,2,3, \cdots .
\end{aligned}
$$

Consider the matrix $C_{e}$. If $q$ is a positive integer, then

$$
\begin{aligned}
\sum_{p=1}^{\infty}\left|c_{q p} e_{p}\right| & =\sum_{p=1}^{i_{1}}\left|c_{q p} e_{p}\right|+\sum_{t=1}^{\infty}\left(\sum_{p=i_{t}+1}^{i_{t+1}}\left|c_{q p} e_{p}\right|\right) \\
& <K+\sum_{t=1}^{\infty}\left(2^{t} \cdot \sum_{p=i_{t}+1}^{i_{t+1}}\left|c_{q p}\right|\right) \\
& \leqq K+\sum_{t=1}^{\infty} 2^{t} / 4^{t} \\
& =K+1 .
\end{aligned}
$$

Let $\left\{r_{p}\right\}$ be an increasing sequence of positive integers such that

$$
\sum_{p=r_{n}+1}^{\infty}\left|a_{p} b_{p}\right|<1 / 4^{n} .
$$

Define

$$
\begin{aligned}
& f_{p}=1, p=1,2, \cdots, r_{1}, \\
& f_{p}=2^{q}, r_{q}<p \leqq r_{q+1}, q=1,2,3, \cdots .
\end{aligned}
$$

Then

$$
\begin{aligned}
\sum_{p=1}^{\infty}\left|a_{p} b_{p} f_{p}\right| & =\sum_{p=1}^{r_{1}}\left|a_{p} b_{p} f_{p}\right|+\sum_{q=1}^{\infty}\left(\sum_{p=r_{q}+1}^{r_{q+1}}\left|a_{p} b_{p} f_{p}\right|\right) \\
& \leqq M+\sum_{q=1}^{\infty}\left(2^{q} \cdot \sum_{p=r_{q}+1}^{r_{q+1}}\left|a_{p} b_{p}\right|\right) \\
& <M+\sum_{q=1}^{\infty} 2^{q} / 4^{q} .
\end{aligned}
$$

Let $g_{p}=\min \left[e_{p}, f_{p}\right], p=1,2,3, \cdots$. Then $g_{p} \rightarrow \infty$ as $p \rightarrow \infty$. Thus $b<b g$. If $n$ is a positive integer, then

$$
\begin{aligned}
\sum_{p=1}^{\infty}\left|a_{n p} b_{p} g_{p}\right| & \leqq \sum_{p=1}^{\infty}\left|c_{n p} g_{p}\right|+\sum_{p=1}^{\infty}\left|a_{p} b_{p} g_{p}\right| \\
& \leqq \sum_{p=1}^{\infty}\left|c_{n p} e_{p}\right|+\sum_{p=1}^{\infty}\left|a_{p} b_{p} f_{p}\right| \\
& <K+1+M+1 .
\end{aligned}
$$

Therefore the matrix $A_{b g}$ sums every null sequence. Thus if $b<b^{\prime}<b g$, then $A$ is c.p.o. [ $\left.b^{\prime}\right]$. The existence of a sequence $b^{\prime}$ such that $b<b^{\prime}<b g$ follows from the lemma. This completes the proof of the theorem. 
REMARK 4. We note that the matrix $A$, defined by $a_{p q}=1$ if $p \neq q, a_{p q}=2^{p-1}$ if $p=q$, has a maximal interval $(-, b)$ over which it is convergence preserving. For example $b=\left\{1 / 2^{p-1}\right\}$.

On the other hand, the matrix $A$, defined by $a_{p q}=0$ if $q>p$, $a_{p q}=1$ if $p \geqq q$, has no such maximal interval. This is easily shown by supposing that $(-, b)$ is a maximal summability interval for $A$. Then $A_{b}$ is c.p.o. $C_{0}$ and hence satisfies the $S-T$ conditions. Thus $\sum_{p=1}^{\infty}\left|b_{p}\right|$ converges. It is easy to find $c \in S_{0}$ such that $b<c$ and $\sum_{p=1}^{\infty}\left|c_{p}\right|$ converges. Thus $A_{c}$ satisfies the $S-T$ conditions and hence is c.p.o. $C_{0}$. Therefore $A$ is c.p.o. $(-, c)$.

It is easy to show that if there exist numbers $r$ and $R$ such that $0<r<\left|a_{p q}\right|<R, p, q=1,2,3, \cdots$, then $A=\left(a_{p q}\right)$ has no maximal summability interval. The proof will be omitted.

REMARK 5. Let $\Delta$ be a chain in $S_{0}$ unbounded above. If $a \in \Delta$, let $a^{\prime}=\left\{a_{1}, 1 / 2, a_{2}, 1 / 4, a_{3}, 1 / 8, \cdots\right\}$. Let $\Delta^{\prime}=\left\{a^{\prime}: a \in \Delta\right\}$. Then $\Delta^{\prime}$ is a chain in $S_{0}$ which is unbounded above. Let $A=\left(a_{p q}\right)$ be defined by $a_{p q}=1 / 2^{n}$ if $q=2 n-1, a_{p q}=1$ if $q$ is an even integer. Clearly if $a^{\prime} \in \Delta^{\prime}$, then $A$ is c.p.o. [a']. But $A$ is not c.p.o. $C_{0}$.

THEOREM 2. If $A$ is c.p.o. each of the sets $\left[b^{(1)}\right],\left[b^{(2)}\right],\left[b^{(3)}\right], \cdots$, then there exists $d \in S_{0}$ such that $b^{(p)}<d, p=1,2,3, \cdots$, and $A$ is c.p.o. $[d]$.

Proof. By Theorem 1 we can find $t^{(n)}$ in $S_{0}$ such that $t^{(n)}>b^{(n)}$ and $A$ is c.p.o. $\left[t^{(n)}\right], n=1,2,3, \ldots$. If $n$ is a positive integer, let $\alpha^{(n)} \in\left[t^{(n)}\right]$ such that $0<\alpha_{p}^{(n)}<1, p=1,2,3, \cdots$. If $n$ is a positive integer, let $M_{n}$ be a number which exceeds $\sum_{q=1}^{\infty}\left|a_{p q} \alpha_{q}^{(n)}\right|, p=1,2,3, \cdots$. If $n$ is a positive integer, let

$$
\beta_{p}^{(n)}=\frac{\alpha_{p}^{(n)}}{2^{n}\left[M_{n}+1\right]}, \quad p=1,2,3, \cdots
$$

If $p$ is a positive integer, let $c_{p}=\sum_{n=1}^{\infty} \beta_{p}^{(n)}$. We wish to show that $c \in S_{0}$. Let $\mu>0$, and let $k$ be a positive integer such that $2^{-k}<\mu / 2$. Let $R$ be a positive integer such that if $q>R$, then $\beta_{q}^{(p)}<\mu / 2^{k+1}$, $p=1,2, \cdots, k$. Then if $n>R$, we have

$$
c_{n}=\sum_{p=1}^{\infty} \beta_{n}^{(p)}=\sum_{p=1}^{k} \beta_{n}^{(p)}+\sum_{p=k+1}^{\infty} \beta_{n}^{(p)}<\mu / 2+2^{-k}<\mu .
$$

Thus $c \in S_{0}$. If $q$ is a positive integer, then, using the double sum theorem, we have 


$$
\begin{aligned}
\sum_{p=1}^{\infty}\left|a_{q p} c_{p}\right| & =\sum_{p=1}^{\infty}\left|a_{q p}\right|\left(\sum_{n=1}^{\infty} \beta_{p}^{(n)}\right) \\
& =\sum_{p=1}^{\infty} \sum_{n=1}^{\infty}\left|a_{q p}\right| \beta_{p}^{(n)} \\
& =\sum_{n=1}^{\infty} \sum_{p=1}^{\infty}\left|a_{q p}\right| \beta_{p}^{(n)} \\
& =\sum_{n=1}^{\infty} \sum_{p=1}^{\infty}\left|a_{q p}\right| \cdot \frac{\alpha_{p}^{(n)}}{2^{n}\left[M_{n}+1\right]} \\
& =\sum_{n=1}^{\infty} \frac{1}{2^{n}\left[M_{n}+1\right]} \sum_{p=1}^{\infty}\left|a_{q p}\right| \alpha_{p}^{(n)} \\
& <\sum_{n=1}^{\infty} 2^{-n} .
\end{aligned}
$$

Thus $A_{c}$ sums every null sequence. Therefore $A$ sums every sequence $x \in S_{0}$ such that $x<c$. We note that if $n$ is a positive integer, then $c_{p} / \beta_{p}^{(n)}>1, p=1,2,3, \cdots$. Thus if $n$ is a positive integer, then

$$
\lim _{p \rightarrow \infty}\left|\frac{c_{p}}{b_{p}^{(n)}}\right|=\lim _{p \rightarrow \infty} \frac{c_{p}}{\beta_{p}^{(n)}} \cdot \frac{\beta_{p}^{(n)}}{t_{p}^{(n)}} \cdot \frac{t_{p}^{(n)}}{\left|b_{p}^{(n)}\right|}=\infty .
$$

Hence $b^{(n)}<c, n=1,2,3, \cdots$. By the lemma, there exists $d \in S_{0}$ such that $b^{(n)}<d<c, n=1,2,3, \cdots . \quad A$ is c.p.o. [d] since $d<c$ and $A$ sums every sequence $x \in S_{0}$ such that $x<c$. This completes the proof of the theorem.

Corollary. Suppose $M$ is a countable set of matrices and $L$ is a countable subset of $E_{0}$ such that if $A \in M$ and $[b] \in L$, then $A$ is c.p.o. [b]. Then there exists $[c] \in E_{0}$ such that if $A \in M$ and $[b] \in L$, then $[b]<^{\prime}[c]$ and $A$ is c.p.o. [c].

Proof. The proof will be for the case that both $M$ and $L$ are infinite sets. Let $M=\left\{A^{(1)}, A^{(2)}, A^{(3)}, \cdots\right\}$ and $L=\left\{\left[b^{(1)}\right],\left[b^{(2)}\right],\left[b^{(3)}\right], \cdots\right\}$. By Theorem 2, if $p$ is a positive integer, there exists $c^{(p)} \in S_{0}$ such that $b^{(n)}<c^{(p)}, n=1,2,3, \cdots$, and $A^{(p)}$ is c.p.o. $\left[c^{(p)}\right]$. Let $L^{\prime}=$ $\left\{\left[c^{(1)}\right],\left[c^{(2)}\right],\left[c^{(3)}\right], \cdots\right\}$. By the lemma, there exists $c \in S_{0}$ such that if $b^{(s)} \in L$ and $c^{(t)} \in L^{\prime}$, then $b^{(s)}<c<c^{(t)}$. If $j$ is a positive integer, then by Remark $2^{\prime}, A^{(j)}$ is c.p.o. $[c]$ since $A^{(j)}$ is c.p.o. $\left[c^{(j)}\right]$ and $[c] \subset\left(-, c^{(j)}\right]$. This completes the proof.

Received January 31, 1967.

North TeXas State University 


\section{PACIFIC JOURNAL OF MATHEMATICS}

\section{EDITORS}

\section{H. ROYDEN}

Stanford University

Stanford, California

\section{J. P. Jans}

University of Washington

Seattle, Washington 98105

\section{J. DUGUNDJI}

Department of Mathematics

Rice University

Houston, Texas 77001

RICHARD ARENS

University of California

Los Angeles, California 90024

\section{ASSOCIATE EDITORS}
E. F. BeCKenbaCH
B. H. NeumanN
F. WOLF
K. YosIDA

\section{SUPPORTING INSTITUTIONS}

UNIVERSITY OF BRITISH COLUMBIA CALIFORNIA INSTITUTE OF TECHNOLOGY UNIVERSITY OF CALIFORNIA MONTANA STATE UNIVERSITY UNIVERSITY OF NEVADA NEW MEXICO STATE UNIVERSITY OREGON STATE UNIVERSITY UNIVERSITY OF OREGON OSAKA UNIVERSITY UNIVERSITY OF SOUTHERN CALIFORNIA
STANFORD UNIVERSITY UNIVERSITY OF TOKYO UNIVERSITY OF UTAH WASHINGTON STATE UNIVERSITY UNIVERSITY OF WASHINGTON

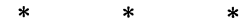

AMERICAN MATHEMATICAL SOCIETY CHEVRON RESEARCH CORPORATION TRW SYSTEMS

NAVAL ORDNANCE TEST STATION 


\section{Pacific Journal of Mathematics}

\section{Vol. 24, No. $1 \quad$ May, 1968}

Harry P. Allen, Lie algebras of type $D_{4}$ over algebraic number fields ...... 1

Charles Ballantine, Products of positive definite matrices. II............ 7

David W. Boyd, The spectral radius of averaging operators ............ 19

William Howard Caldwell, Hypercyclic rings ................... 29

Francis William Carroll, Some properties of sequences, with an application

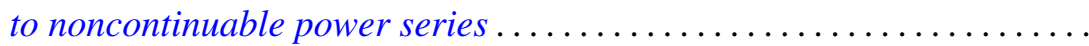

David Fleming Dawson, Matrix summability over certain classes of

sequences ordered with respect to rate of convergence ........... 51

D. W. Dubois, Second note on David Harrison's theory of preprimes. . . . . 57

Edgar Earle Enochs, A note on quasi-Frobenius rings.............. 69

Ronald J. Ensey, Isomorphism invariants for Abelian groups modulo bounded groups ................................ 71

Ronald Owen Fulp, Generalized semigroup kernels ................ 93

Bernard Robert Kripke and Richard Bruce Holmes, Interposition and approximation ................................. 103

Jack W. Macki and James Sai-Wing Wong, Oscillation of solutions to second-order nonlinear differential equations ..................

Lothrop Mittenthal, Operator valued analytic functions and generalizations

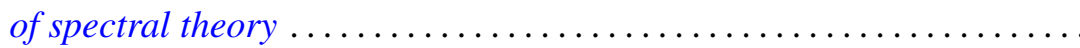

T. S. Motzkin and J. L. Walsh, A persistent local maximum of the pth power deviation on an interval, $p<1 \ldots \ldots \ldots \ldots \ldots \ldots \ldots \ldots \ldots \ldots . \ldots \ldots$

Jerome L. Paul, Sequences of homeomorphisms which converge to homeomorphisms ...........................

Maxwell Alexander Rosenlicht, Liouville's theorem on functions with elementary integrals.

Joseph Goeffrey Rosenstein, Initial segments of degrees .

$\mathrm{H}$. Subramanian, Ideal neighbourhoods in a ring ............

Dalton Tarwater, Galois cohomology of abelian groups . .

James Patrick Williams, Schwarz norms for operators ... .

Raymond Y. T. Wong, A wild Cantor set in the Hilbert cube. 